\title{
ADAPTED SETS OF MEASURES AND INVARIANT FUNCTIONALS ON $L^{p}(G)$
}

\author{
RODNEY NILLSEN
}

\begin{abstract}
Let $G$ be a locally compact group. If $G$ is compact, let $L_{0}^{p}(G)$ denote the functions in $L^{p}(G)$ having zero Haar integral. Let $M^{1}(G)$ denote the probability measures on $G$ and let $\mathscr{P}^{1}(G)=M^{1}(G) \cap L^{1}(G)$. If $S \subseteq$ $M^{1}(G)$, let $\Delta\left(L^{p}(G), S\right)$ denote the subspace of $L^{p}(G)$ generated by functions of the form $f-\mu * f, f \in L^{p}(G), \mu \in S$. If $G$ is compact, $\Delta\left(L^{p}(G), S\right) \subseteq$ $L_{0}^{p}(G)$. When $G$ is compact, conditions are given on $S$ which ensure that for some finite subset $F$ of $S, \Delta\left(L^{p}(G), F\right)=L_{0}^{p}(G)$ for all $1<p<\infty$. The finite subset $F$ will then have the property that every $F$-invariant linear functional on $L^{p}(G)$ is a multiple of Haar measure. Some results of a contrary nature are presented for noncompact groups. For example, if $1 \leq p \leq \infty$, conditions are given upon $G$, and upon subsets $S$ of $M^{1}(G)$ whose elements satisfy certain growth conditions, which ensure that $L^{p}(G)$ has discontinuous, $S$-invariant linear functionals. The results are applied to show that for $1 \leq p \leq$ $\infty, L^{p}(\mathbb{R})$ has an infinite, independent family of discontinuous translation invariant functionals which are not $\mathscr{P}^{1}(\mathbb{R})$-invariant.
\end{abstract}

\section{INTRODUCTION}

On the circle group $\mathbf{T}$ let $L_{0}^{2}(\mathbf{T})=\left\{f: f \in L^{2}(\mathbf{T})\right.$ and $\left.\int_{\mathbf{T}} f=0\right\}$. If $\phi \geq 0$ on $\mathbf{T}, \int_{\mathbf{T}} \phi=1$ and $\hat{\phi}$ denotes the Fourier Transform of $\phi$, then $|\hat{\phi}|$ is bounded away from one on $\mathbf{Z} \cap\{0\}^{c}$. Consequently, the linear operator defined on $\ell^{2}\left(Z \cap\{0\}^{c}\right)$ by $d \rightarrow d-\hat{\phi} d$ is bounded and invertible. It then follows from the Riesz-Fischer Theorem that $L_{0}^{2}(\mathbf{T})=\left\{f-\phi * f: f \in L^{2}(\mathbf{T})\right\}$. It is an easy consequence of this that any $\phi$-invariant linear functional on $L^{2}(\mathbf{T})$ is a multiple of Lebesgue measure and is therefore continuous.

One purpose of this paper is to extend these results in several ways. The circle group is replaced by a general compact group $G, L_{0}^{2}(\mathbf{T})$ is replaced by $L_{0}^{p}(G)=\left\{f: f \in L^{p}(G)\right.$ and $\left.\int_{G} f=0\right\}$ and instead of considering convolution by a single probability measure in $L^{1}(\mathbf{T})$, as above, convolutions by elements of some given family of probability measures on $G$ are considered simultaneously. Here, the main result is the following.

Theorem. Let $G$ be a compact group with dual $\hat{G}$ and let $S$ be a subset of $M^{1}(G)$ such that (i) the elements of $S$ are not simultaneously supported by a

Received by the editors May 1, 1989.

1980 Mathematics Subject Classification (1985 Revision). Primary 43A15. 
proper closed subgroup of $G$, and (ii) some average of elements of $S$ has a Fourier transform whose norm is bounded away from one on the complement of some finite subset of $\hat{G}$. Then there exist $\mu_{1}, \mu_{2}, \ldots, \mu_{n} \in S$ so that for all $1<p<\infty$,

$$
L_{0}^{p}(G)=\left\{\sum_{i=1}^{n}\left(f_{i}-\mu_{i} * f_{i}\right): f_{1}, f_{2}, \ldots, f_{n} \in L^{p}(G)\right\} .
$$

Also, any linear functional on $L^{p}(G)$ which is $\mu_{i}$-invariant for each $1 \leq i \leq n$, is a multiple of Haar measure and is therefore continuous.

This type of result is an analogue for convolutions of earlier results which have been obtained for translations in $L^{p}(G)$. Among these is the result of G. Meisters and W. Schmidt [6] according to which every translation invariant linear form on $L^{2}(G)$ is continuous, for any compact connected abelian group $G$. On the other hand, Meisters showed in [7] that if $G$ is compact and disconnected, or if $G$ is noncompact, $L^{2}(G)$ may have discontinuous translation invariant linear functionals. If $G$ is a given compact abelian group whose component of the identity is $C, \mathrm{~L}$. Bagget and G. Meisters [8, p. 436] proved that if every translation invariant linear functional on $L^{2}(G)$ is continuous then $G / C$ has a finitely generated dense subgroup, and the converse of this statement was proved by B. Johnson in [4]. More recently, J. Bourgain [1] has proved that for $1<p<\infty$, every translation invariant linear form on $L^{p}(\mathbf{T})$ is continuous.

In [16], G. Woodward proved that for a noncompact, $\sigma$-compact group, $L^{1}(G)$ admits translation invariant, discontinuous linear functionals. If it is further assumed that $G$ is amenable, he proved that for $1<p \leq \infty, L^{p}(G)$ also admits translation invariant, discontinuous linear functionals. On the other hand, if $G$ is not amenable, $G$. Willis [15] has proved that there are no nonzero translation invariant linear functionals on $L^{p}(G)$ for $1<p \leq \infty$.

A second purpose of this paper is to obtain analogues of these results of Woodward where translations on the group are replaced by convolutions by measures in $M^{1}(G)$ which satisfy certain growth conditions. These results are then applied to show that for each $1 \leq p \leq \infty, L^{p}(\mathbb{R})$ has an infinite, linearly independent family of translation invariant, discontinuous linear functionals which are not $\mathscr{P}^{1}(\mathbb{R})$-invariant. This is proved by showing that the subspace of $L^{p}(\mathbb{R})$ spanned by $\left\{f-\delta_{g} * f: f \in L^{p}(\mathbb{R})\right.$ and $\left.g \in \mathbb{R}\right\}$ is both contained and has infinite codimension in the subspace of $L^{p}(\mathbb{R})$ spanned by $\left\{f-\phi * f: f \in L^{p}(\mathbb{R})\right.$ and $\left.\phi \in \mathscr{P}^{1}(\mathbb{R})\right\}$. Some notation to be used in the paper now follows.

Let $M(G)$ denote the regular Borel probability measures on $G$, let $M^{1}(G)$ denote the probability measures in $M(G)$ and let $\mathscr{P}^{1}(G)=M^{1}(G) \cap L^{1}(G)$. If $\mu \in M(G), \tilde{\mu}$ is defined in $M(G)$ by $\tilde{\mu}(A)=\overline{\mu\left(A^{-1}\right)}$. Let $S \subseteq M^{1}(G)$ and let $X$ be a Banach space of functions on $G$ or let $X$ be $L^{p}(G)$ for some $1 \leq p \leq \infty$. Then $X$ is said to be $S$-invariant if for all $\mu \in S$ and $f \in X$, the 
convolution $\mu * f$ is defined and is in $X$. In this case let

$$
\Delta(X, S)=\left\{\sum_{i=1}^{n}\left(f_{i}-\mu_{i} * f_{i}\right): n \in \mathbb{N}, \mu_{i} \in S \text { and } f_{i} \in X \text { for } 1 \leq i \leq n\right\} .
$$

If $S=\left\{\delta_{g}: g \in G\right\}$ and $X$ is $S$-invariant, then $X$ is said to be translation invariant, and $\Delta(X, S)$ is denoted by $\Delta(X, G)$. Let $X^{\prime}$ denote the algebraic dual of $X$, and let $X$ be $S$-invariant. Then an element $T$ of $X^{\prime}$ is said to be $S$-invariant if $T(\mu * f)=T(f)$ for all $\mu \in S$ and $f \in X$. Clearly, $T$ is $S$-invariant if and only if $T=0$ on $\Delta(X, S)$. If $X$ is translation invariant, a functional $T \in X^{\prime}$ is said to be translation invariant if it is $\left\{\delta_{g}\right\}$-invariant for all $g \in G$. The set of all $S$-invariant functionals in $X^{\prime}$ is denoted by $I(X, S)$, and the set of all translation invariant functionals in $X^{\prime}$ is denoted by $I(X, G)$. Of course, $I(X, G)=I\left(X,\left\{\delta_{g}: g \in G\right\}\right)$.

A particular left invariant Haar measure on the locally compact group $G$ will be denoted by $\lambda$. The bounded continuous complex-valued functions on $G$ will be denoted by $C(G)$, the functions in $C(G)$ which vanish at infinity will be denoted by $C_{0}(G)$, and the left uniformly continuous functions in $C(G)$ will be denoted by $C U(G)$. Each of the spaces $C_{0}(G), C U(G), C(G)$ and $L^{p}(G)$ for $1 \leq p \leq \infty$ is $M^{1}(G)$-invariant. The identity element of $G$ is denoted by $e$.

\section{THE GENERAL SETTING}

Let $S$ be a subset of $M^{1}(G)$ and let $X$ be a space $L^{p}(G)$ for some $1 \leq p \leq$ $\infty$, or let $X$ be an $S$-invariant Banach space of complex valued functions on $G$.

Proposition 1. If $\Delta(X, S)$ is not closed in $X$, there are discontinuous $S$-invariant functionals on $X$.

Proof. With trivial changes, this may be proved along the lines of $[7$, Theorem 1] (see also [16, Theorem A]).

Lemma 1. Let $S, S_{1}$ and $S_{2}$ be subsets of $M^{1}(G)$, and let $X$ be invariant under $S, S_{1}$ and $S_{2}$. Then the following hold:

(i) $\Delta(X, S)=\bigcap\{$ Kernel of $T: T \in I(X, S)\}$, and

(ii) $I\left(X, S_{1}\right) \subseteq I\left(X, S_{2}\right)$ if and only if $\Delta\left(X, S_{1}\right) \supseteq \Delta\left(X, S_{2}\right)$.

Proof. It is obvious that if $T \in X^{\prime}$, then $T \in I(X, S)$ if and only if $T=0$ on $\Delta(X, S)$. Also, if $h \in X \cap \Delta(X, S)^{c}$, there is $T_{0} \in X^{\prime}$ so that $T_{0}(h) \neq 0$ but $T_{0}=0$ on $\Delta(X, S)$. Then $T_{0} \in I(X, S)$ but $h$ does not belong to the kernel of $T_{0}$. Conclusions (i) and (ii) follow from these observations.

Proposition 2. Let $S_{1}$ and $S_{2}$ be subsets of $M^{1}(G)$ such that for some $\nu \in S_{1}$, $\nu * S_{2} \subseteq S_{1}$. Then $I\left(X, S_{1}\right) \subseteq I\left(X, S_{2}\right)$ and $\Delta\left(X, S_{2}\right) \subseteq \Delta\left(X, S_{1}\right)$. 
Proof. Let $T \in I\left(X, S_{1}\right), \mu \in S_{2}$ and $f \in X$. Then $T(\mu * f)=T(\nu * \mu * f)=$ $T(f)$, as $\nu * \mu \in S_{1}$. Hence, $T \in I\left(X, S_{2}\right)$ so that $I\left(X, S_{1}\right) \subseteq I\left(X, S_{2}\right)$. The remainder follows from (ii) of Lemma 1.

Corollary. Let $1 \leq p \leq \infty$. Then $\Delta\left(L^{p}(G), G\right) \subseteq \Delta\left(L^{p}(G), \mathscr{P}^{1}(G)\right)$ and $I\left(L^{p}(G), G\right) \supseteq I\left(L^{p}(G), \mathscr{P}^{1}(G)\right)$.

Proof. If $a \in G$ and $\phi \in \mathscr{P}^{1}(G)$, then $\phi * \delta_{a} \in \mathscr{P}^{1}(G)$. Hence Proposition 1 applies.

Definitions. A nonempty subset $S$ of $M^{1}(G)$ is said to be adapted if $\mu(A)=1$ for all $\mu \in S$ implies that the group generated by $A$ is dense in $G$. This is equivalent to requiring that there is no proper closed subgroup of $G$ which supports all elements of $S$. A single measure $\mu \in S$ is said to be adapted if $\{\mu\}$ is adapted.

If $G$ is noncompact, a family $\left(A_{\alpha}\right)_{\alpha \in I}$ of subsets of $G$ is called dispersed if for each $n \in \mathbb{N}$, and each compact subset $K$ of $G$, there are $\alpha_{1}, \alpha_{2}, \ldots, \alpha_{n} \in$ $I$ such that $A_{\alpha_{i}} K \cap A_{\alpha_{j}} K=\varnothing$ for all $1 \leq i<j \leq n$.

Proposition 3. Let $G$ be $\sigma$-compact and let $S$ be a nonempty subset of $M^{1}(G)$. Then the following hold:

(i) if $G$ is compact, $S$ is adapted and $1<p<\infty$, then $\Delta\left(L^{p}(G), S\right)$ is dense in $L_{0}^{p}(G)$, and the only continuous $S$-invariant linear functionals on $L^{p}(G)$ are the multiples of Haar measure,

(ii) if $G$ is not compact, $S$ is adapted and $1<p<\infty$, then $\Delta\left(L^{p}(G), S\right)$ is dense in $L^{p}(G)$, and the only continuous $S$-invariant linear functional on $L^{p}(G)$ is 0 ,

(iii) if $S$ is right translation invariant, then $\Delta\left(L^{1}(G), S\right)$ is dense in $L_{0}^{1}(G)$ and the only continuous $S$-invariant linear functionals on $L^{1}(G)$ are the multiples of Haar measure, and

(iv) - if $G$ is noncompact and if the supports of the measures in $S$ form a dispersed family in $G$, then $\Delta\left(C_{0}(G), S\right)$ is dense in $C_{0}(G)$.

Proof. The first statements in each of (i), (ii) and (iii) are contained in Theorem 3 of [9]. The proofs of the remaining statements in (i) and (ii) are similar to the following one for (iii). Choose $f \in L^{1}(G)$ so that $\int_{G} f d \lambda \neq 0$. Then for $g \in L^{1}(G)$,

$$
g=\left(\int_{G} g d \lambda\right)\left(\int_{G} f d \lambda\right)^{-1} f+\left[g-\left(\int_{G} g d \lambda\right)\left(\int_{G} f d \lambda\right)^{-1} f\right] .
$$

The function in the square brackets is in $L_{0}^{1}(G)$. Hence, if $L$ is a continuous $S$-invariant linear functional on $L^{1}(G)$, we have $L=0$ on $L_{0}^{1}(G)$ by the first part of (iii), so that $L(g)=\left(\int_{G} f d \lambda\right)^{-1} L(f) \int_{G} g d \lambda$, for all $g \in L^{1}(G)$.

To prove (iv), let $f \in C_{0}(G)$ and $n \in \mathbb{N}$. Let $K$ be a compact subset of $G$ so that $|f(x)|<n^{-1}$, for all $x \in G \cap K^{c}$. Then choose $\mu_{1}, \mu_{2}, \ldots, \mu_{n} \in S$ so that 
if $A_{1}, A_{2}, \ldots, A_{n}$ respectively denote their supports, then $A_{i} K \cap A_{j} K=\varnothing$, for $i \neq j$. Then if $x \notin A_{i} K$,

$$
\left|\left(\mu_{i} * f\right)(x)\right| \leq \int_{A_{i}}\left|f\left(s^{-1} x\right)\right| d \mu_{i}(s) \leq n^{-1} .
$$

Hence, $\left|\left(\sum_{i=1}^{n} \mu_{i} * f\right)(x)\right| \leq 1$, if $x \notin \bigcup_{i=1}^{n} A_{i} K$. On the other hand, if $x \in$ $\bigcup_{i=1}^{n} A_{i} K, x \in A_{j} K$ for a unique $j$, so in this case $\left|\left(\sum_{i=1}^{n} \mu_{i} * f\right)(x)\right| \leq$ $(n-1) n^{-1}+\|f\|_{\infty}$. Hence, $\left\|\sum_{i=1}^{n} \mu_{i} * f\right\|_{\infty} \leq 1+\|f\|_{\infty}$. If $\nu \in C_{0}(G)^{*}$ is $S$-invariant, we now have

$$
|\nu(f)|=\left|\nu\left(n^{-1}\left(\sum_{i=1}^{n} \mu_{i} * f\right)\right)\right| \leq n^{-1}\|\nu\|\left(1+\|f\|_{\infty}\right),
$$

true for all $n$, so $\nu(f)=0$. Hence, if $\nu \in C_{0}(G)^{*}$ and $\nu=0$ on $\Delta\left(C_{0}(G), S\right)$, then $\nu=0$. It follows that $\Delta\left(C_{0}(G), S\right)$ is dense in $C_{0}(G)$.

Remark. The proof of (iv) is an adaptation of [16, Lemma 1(i)], which applies when $S=\left\{\delta_{g}: g \in G\right\}$ (see also [10, pp. 237-238]).

\section{RESULTS ON COMPACT GROUPS}

If $G$ is a locally compact group, let $\hat{G}$ denote the dual object of $G$, and let $l$ denote the trivial representation in $\hat{G}$. If $\sigma \in \hat{G}$, we regard $\sigma$ as a particular continuous unitary representation of $G$ in $B\left(H_{\sigma}\right)$, the bounded linear operators on a Hilbert space $H_{\sigma}$ whose dimension is denoted by $d_{\sigma}$ and whose unit ball is denoted by $U_{\sigma}$. If $\mu \in M(G)$ and $\sigma \in \hat{G}, \hat{\mu}(\sigma) \in B\left(H_{\sigma}\right)$ is given by the equation

$$
\langle\hat{\mu}(\sigma) u, v\rangle=\int_{G}\langle\sigma(t) u, v\rangle d \mu(t), \quad \text { for all } u, v \in H_{\sigma} .
$$

If $S \subseteq M^{1}(G)$, let $H(S)$ be defined by

$$
H(S)=\left\{n^{-1}\left(\sum_{i=1}^{n} \mu_{i}\right): n \in \mathbb{N} \text { and } \mu_{i} \in S \text { for all } 1 \leq i \leq n\right\} .
$$

Then $S \subseteq H(S)$.

Lemma 2. Let $G$ be a compact group and let $S$ be a subset of $M^{1}(G)$. Then the following hold:

(i) If $S$ is adapted, for each $\sigma \in \hat{G}$ with $\sigma \neq l$, there is $\nu \in H(S)$ such that $I_{\sigma}-\hat{\nu}(\sigma)$ is invertible on $H_{\sigma}$, and

(ii) if $\{\tilde{\mu} * \mu: \mu \in S\}$ is adapted, for each $\sigma \in \hat{G}$ with $\sigma \neq l$, there is $\nu \in H(S)$ such that $\|\hat{\nu}(\sigma)\|<1$.

Proof. (i) Let $S$ be adapted and let $\sigma \in \hat{G}$ with $\sigma \neq \imath$. Now let $u \in H_{\sigma}$, $\|u\|=1$ and $\hat{\mu}(\sigma) u=u$ for all $\mu \in S$. Then

$$
\int_{G}(1-\langle(\sigma(x))(u), u\rangle) d \mu(x)=\int_{G}(\langle u, u\rangle-\langle(\sigma(x))(u), u\rangle) d \mu(x)=0,
$$


for all $\mu \in S$. As $\mu \geq 0$, and as $x \rightarrow\langle(\sigma(x))(u), u\rangle$ is continuous on $G$, we deduce that

$$
1=\langle u, u\rangle=\langle(\sigma(x))(u), u\rangle,
$$

for all $x$ belonging to the support of $\mu$, for any $\mu \in S$. Thus, for any such $x$, $(\sigma(x))(u)$ is a multiple of $u$. Using (3.1), it now follows that $(\sigma(x))(u)=u$, for all $x$ belonging to the support of any $\mu \in S$. As $S$ is adapted, $(\sigma(x))(u)=u$ for all $x \in G$, which contradicts the fact that $\sigma \neq l$ and $\sigma$ is irreducible.

It now follows that if $\sigma \in \hat{G} \cap\{l\}^{c}$,

$$
\bigcap\left\{\text { Kernel of } I_{\sigma}-\hat{\mu}(\sigma): \mu \in S\right\}=\{0\} \text {. }
$$

Each kernel of $I_{\sigma}-\hat{\mu}(\sigma)$ is closed. Also, $U_{\sigma}$ is compact, as compactness of $G$ implies $H_{\sigma}$ is finite dimensional. Hence there are $\mu_{1}, \ldots, \mu_{n} \in S$ so that

$$
\bigcap\left\{\text { Kernel of } I_{\sigma}-\hat{\mu}_{i}(\sigma): i=1,2, \ldots, n\right\}=\{0\} .
$$

Let $\nu=n^{-1}\left(\sum_{i=1}^{n} \mu_{i}\right) \in H(S)$. Then if $\left(I_{\sigma}-\hat{\nu}(\sigma)\right)(u)=0$, for some $u \in H_{\sigma}$ with $\|u\|=1$, we have

$$
u=n^{-1}\left(\sum_{i=1}^{n} \hat{\mu}_{i}(\sigma) u\right) .
$$

As $u$ is an extreme point of $U_{\sigma}$, and as $\hat{\mu}(\sigma) U_{\sigma} \subseteq U_{\sigma}$ for all $\mu \in M^{1}(G)$, it follows that $u=\hat{\mu}_{i}(\sigma) u$, for all $1 \leq i \leq n$. Hence,

$$
u \in \bigcap\left\{\text { Kernel of } I_{\sigma}-\hat{\mu}_{i}(\sigma): 1 \leq i \leq n\right\}=\{0\},
$$

so $u=0$. Hence $I_{\sigma}-\hat{\nu}(\sigma)$ is injective on $H_{\sigma}$. As $H_{\sigma}$ is finite dimensional, $I_{\sigma}-\hat{\nu}(\sigma)$ is invertible on $H_{\sigma}$. This proves (i).

(ii) Let $\{\tilde{\mu} * \mu: \mu \in S\}$ be adapted and let $\sigma \in \hat{G} \cap\{l\}^{c}$. Then as in the proof of (i) it follows that there are $\mu_{1}, \ldots, \mu_{n} \in S$ so that

$$
\bigcap\left\{\text { Kernel of }\left(\left(\tilde{\mu}_{i} * \mu_{i}\right)^{\wedge}(\sigma)-I_{\sigma}\right): 1 \leq i \leq n\right\}=\{0\} .
$$

Hence, if $u \in H_{\sigma}$ and $\|u\|=1$, then for some $i \in\{1,2, \ldots, n\},\left\|\hat{\mu}_{i}(\sigma) u\right\|<$ 1. As $H_{\sigma}$ is finite dimensional, $U_{\sigma}$ is norm compact and it follows that $\left\|n^{-1} \cdot\left(\sum_{i=1}^{n} \hat{\mu}_{i}(\sigma)\right)\right\|<1$. Hence, if we let $\nu=n^{-1}\left(\sum_{i=1}^{n} \mu_{i}\right), \nu \in H(S)$ and $\|\hat{\nu}(\sigma)\|<1$.

Lemma 3. Let $S$ be a subset of $M^{1}(G)$. Then if $\{\tilde{\mu} * \mu: \mu \in S\}$ is adapted, $S$ is adapted. Also, if $S$ is adapted, if $S \subset \mathscr{P}^{1}(G)$ and if $e \in \bigcap\{$ support of $\mu: \mu \in S\}$, then $\{\tilde{\mu} * \mu: \mu \in S\}$ is adapted.

Proof. Let $H$ be a closed subgroup of $G$ so that $\mu(H)=1$ for all $\mu \in S$. Then $(\tilde{\mu} * \mu)(H)=1$, for all $\mu \in S$. Hence if $\{\tilde{\mu} * \mu: \mu \in S\}$ is adapted, so too is $S$.

On the other hand, let $H$ be a closed subgroup of $G$ so that $(\tilde{\mu} * \mu)(H)=1$, for all $\mu \in S$. Then for each $\mu \in S, \mu(s H)=1$ for $\mu$-almost all $s \in G$. If $S \subseteq \mathscr{P}^{1}(G), s \rightarrow \mu(s H)$ is continuous on $G$ for all $\mu \in S$. Hence in 
this case $\mu(s H)=1$ for all $s$ in the support of $\mu$, for each $\mu \in S$. If $e \in \bigcap\{$ support of $\mu: \mu \in s\}$, we then have $\mu(H)=1$ for all $\mu \in S$. Thus, under these assumptions, if $S$ is adapted, so too is $\{\tilde{\mu} * \mu: \mu \in S\}$.

Remark. It is not true in general that if $S$ is adapted, so is $\{\tilde{\mu} * \mu: \mu \in S\}$. For example, let $H$ be a proper closed subgroup of $G$ and let $x \in G$ be such that $x H$ is not contained in a proper closed subgroup of $G$. Let $\nu$ be the Haar measure on $H$. Then $\delta_{x} * \nu$ may belong to $\mathscr{P}^{1}(G)$ and in this case $\delta_{x} * \nu$ is adapted. However, $\left(\delta_{x} * \nu\right)^{\sim} *\left(\delta_{x} * \nu\right)=\nu$, which is not adapted.

The following result uses some concepts and terminology from the interpolation theory of linear operators. Definitions of undefined terms and a description of the interpolation method may be found in [5, Chapter IV].

Lemma 4. Let $B$ be a vector space which is the direct sum of two vector subspaces $C$ and $D$. Let $\pi: B \rightarrow C$ be the associated projection. Let $\||\cdot|\|_{0}$ and $\||\cdot|\|_{1}$ be two norms on $B$ which are consistent, and let $\pi$ be bounded in each of these norms. If $X$ is a vector subspace of $B$ and $0<\alpha<1$, let $\||\cdot|\|_{X, \alpha}$ denote the norm obtained on $X$ by interpolating between the restrictions to $X$ of $\|\cdot \cdot\|_{0}$ and $\|\cdot \mid\|_{1}$. Let $\||\cdot|\|_{B, C, \alpha}$ denote the norm on the quotient $B / C$ obtained in the usual way from the norm $\||\cdot|\|_{B, \alpha}$ on $B$. Then the spaces $\left(D,\|\cdot \mid\| \|_{D, \alpha}\right)$, $\left(B / C,\||\cdot|\|_{B, C, \alpha}\right)$ and $\left(D,\||\cdot|\|_{B, \alpha}\right)$ are mutually isomorphic as normed vector spaces.

Proof. The result is surely known, although I have no explicit reference. It can be proved in a routine if tedious manner from the assumptions and the definition of the interpolation procedure that $\left(D,\left|\|\cdot \mid\|_{D, \alpha}\right)\right.$ and $\left(B / C,\||| \cdot \mid\|_{B, C, \alpha}\right)$ are isomorphic. The isomorphism of this latter space with $\left(D,\||\cdot|\|_{B, \alpha}\right)$ is then easily checked.

Theorem 1. Let $G$ be a compact group and let $S$ be an adapted subset of $M^{1}(G)$. Suppose that there exist $0<\delta<1$, a finite subset $F$ of $\hat{G}$ and $\nu \in H(S)$ such that $\| \hat{\nu}\left(\sigma \| \leq \delta\right.$ for all $\sigma \in \hat{G} \cap F^{c}$. Then there exist $\mu_{1}, \mu_{2}, \ldots, \mu_{n} \in S$ so that for each $1<p<\infty$,

$$
L_{0}^{p}(G)=\left\{\sum_{i=1}^{n}\left(f_{i}-\mu_{i} * f_{i}\right): f_{1}, f_{2}, \ldots, f_{n} \in L^{p}(G)\right\} .
$$

Also, if $\psi$ is any linear functional on $L^{p}(G)$, for some $1<p<\infty$, and such that $\psi\left(\mu_{i} * f\right)=\psi(f)$ for all $1 \leq i \leq n$ and $f \in L^{p}(G)$, then $\psi$ is continuous on $L^{p}(G)$ and is a multiple of Haar measure.

Proof. For each $\sigma \in \hat{G}$, let $u_{i j}^{(\sigma)}, 1 \leq i, j \leq d_{\sigma}$, be a set of coordinate functions for $\sigma$. Let $\mathscr{F}_{\sigma}$ (respectively, $\mathscr{F}_{\sigma, k}$ for $1 \leq k \leq d_{\sigma}$ ) be the subspace of $L^{2}(G)$ spanned by $u_{i j}^{(\sigma)}, 1 \leq i, j \leq d_{\sigma}$ (respectively, $u_{i k}^{(\sigma)}$ for $1 \leq i \leq$ $\left.d_{\sigma}\right)$. If $\rho_{1}, \rho_{2}, \ldots, \rho_{d_{\sigma}}$ is an orthonormal basis for $H_{\sigma}$ such that $u_{i j}^{(\sigma)}(x)=$ $\left\langle\sigma(x) \rho_{j}, \rho_{i}\right\rangle$ for all $x \in G$ and all $1 \leq i, j \leq d_{\sigma}$, let $T_{\sigma, k}: \mathscr{F}_{\sigma, k} \rightarrow H_{\bar{\sigma}}$ be 
defined for $1 \leq k \leq d_{\sigma}$ by

$$
T_{\sigma, k}\left(\sum_{i=1}^{d_{\sigma}} c_{i} \sqrt{d_{\sigma}} u_{i k}^{(\sigma)}\right)=\sum_{i=1}^{d_{\sigma}} c_{i} \rho_{i} .
$$

Then $T_{\sigma, k}$ is a linear isometry, which shows that the left regular representation of $G$ on $\mathscr{F}_{\sigma, k}$ is equivalent to $\bar{\sigma}\left[3\right.$, p. 36]. If $\mu \in M(G)$, let $T_{\mu}$ denote the operator obtained by convolution by $\mu$. Then for $\mu \in M(G), T_{\sigma, k} \circ T_{\mu}=$ $\hat{\mu}(\bar{\sigma}) \circ T_{\sigma, k}$ on $\mathscr{F}_{\sigma, k}$, so that $\hat{\mu}(\bar{\sigma})$ corresponds, under this equivalence, to convolution by $\mu$ on $\mathscr{F}_{\sigma, k}$. Hence, $\left\|T_{\mu}\right\|_{\mathscr{F}_{\sigma, k}}=\|\hat{\mu}(\bar{\sigma})\|$, for $\sigma \in \hat{G}$ and $1 \leq$ $k \leq d_{\sigma}$.

If $F$ is a finite subset of $\hat{G}$ and $1 \leq p \leq \infty$, let

$$
L_{F}^{p}(G)=\left\{f: f \in L^{p}(G) \text { and } \int_{G} f h d \lambda=0 \text { for all } h \in \bigcup_{\sigma \in \bar{F}} \mathscr{F}_{\sigma}\right\},
$$

and let $X_{F}(G)=\sum_{\sigma \in \bar{F}} \mathscr{F}_{\sigma}$. Then $X_{F}(G)$ is a finite dimensional (hence closed) subspace of $L^{p}(G)$. If $\mu \in M(G)$, both $L_{F}^{p}(G)$ and $X_{F}(G)$ are invariant under $T_{\mu}$. Now define $\pi: L^{\infty}(G) \rightarrow X_{F}(G)$ by

$$
\pi(f)=\sum_{\sigma \in \bar{F}}\left(\sum_{i, j=1}^{d_{\sigma}}\left(\int_{G} f u_{i j}^{(\sigma)} d \lambda\right) \overline{u_{i j}^{\sigma}}\right) .
$$

Then $\pi$ is a linear projection from $L^{\infty}(G)$ onto $X_{F}(G)$ and its kernel is $L_{F}^{\infty}(G)$.

Let $2<p<\infty$ and let $r$ be chosen so that $p<r<\infty$. Let $\|\cdot\| \|_{0}$ be the restriction to $L^{\infty}(G)$ of the $L^{2}$-norm and let $\|\mid \cdot\| \|_{1}$ be the corresponding restriction of the $L^{r}$-norm. The projection $\pi$ is continuous in each one of these norms, and the norms are consistent. Choose $0<\alpha<1$ so that the norm on $L^{\infty}(G)$ resulting by interpolating between $\|\cdot \cdot\|_{0}$ and $\|\cdot \cdot\|_{1}$ is the $L^{p}$-norm. Now let $S, F, \nu$ and $\delta$ be as given in the theorem. Then $l \in F$. It follows from the Peter-Weyl theorem and the fact that $\left\|T_{\nu}\right\|_{\mathscr{F}_{\sigma, k}}=\|\hat{\nu}(\bar{\sigma})\|$, that if $h \mid A$ denotes the restriction of a function $h$ to a subset of its domain, then

$$
\left\|T_{\nu} \mid L_{F}^{\infty}(G)\right\|_{1\|\cdot \cdot\|_{0}} \leq \delta<1 .
$$

Hence

$$
\begin{aligned}
\left\|T_{\nu} \mid L_{F}^{\infty}(G)\right\|_{\|\mid\| \|_{L_{F}(G), \alpha}^{\infty}} & \leq\left\|T_{\nu}\left|L_{F}^{\infty}(G)\left\|_{\||\cdot|\|_{0}}^{1-\alpha}\right\| T_{\nu}\right| L_{F}^{\infty}(G)\right\|_{\|\| \cdot\|\|_{1}}^{\alpha} \\
& \leq \delta^{1-\alpha}<1 .
\end{aligned}
$$

Thus, $\left(I-T_{\nu}\right) \mid L_{F}^{\infty}(G)$ is bounded and invertible on the completion of $\left(L_{F}^{\infty}(G), \quad\||\cdot|\|_{L_{F}^{\infty}(G), \alpha}\right)$. By taking in Lemma 4 the spaces $L^{\infty}(G), X_{F}(G)$ and $L_{F}^{\infty}(G)$ for $B, C$ and $D$ respectively, we can now deduce that $I-T_{\nu}$ is a bounded invertible operator on $\left(L_{F}^{p}(G),\|\cdot\|_{p}\right)$. 
Let $f \in L_{0}^{p}(G)$. Then we can write $f=f_{0}+\sum_{\sigma \in F} f_{\sigma}$, where $f_{0} \in L_{F}^{p}(G)$ and $f_{\sigma} \in \mathscr{F}_{\bar{\sigma}}$ for all $\sigma \in F\left(f_{l}=0\right)$. Let $g_{0}=\left(I-T_{\nu}\right)^{-1} f_{0} \in L_{F}^{p}(G)$. If $\sigma \in F \cap\{l\}^{c}$, since $S$ is adapted, by Lemma 2 and the remarks above, it follows that there is $\nu_{\sigma} \in H(S)$ so that $I-T_{\nu_{\sigma}}$ is invertible on $\mathscr{F}_{\bar{\sigma}}$. Let $g_{\sigma}=\left(I-T_{\nu_{\sigma}}\right)^{-1} f_{\sigma} \in \mathscr{F}_{\bar{\sigma}}$. We now have

$$
\begin{aligned}
f & =\left(I-T_{\nu}\right) g_{0}+\sum_{\sigma \in F \cap\{l\}^{c}}\left(I-T_{\nu_{\sigma}}\right) g_{\sigma} \\
& =\left(g_{0}-\nu * g_{0}\right)+\sum_{\sigma \in F \cap\{l\}^{c}}\left(g_{\sigma}-\nu_{\sigma} * g_{\sigma}\right) .
\end{aligned}
$$

It should be noted that the choice of $\nu$ and the $\nu_{\sigma}$ is independent of $p$ and $f \in L_{0}^{p}(G)$. This proves (3.2) for $2<p<\infty$ and also contains the proof with $p=2$. If $1<p<2$, an interpolation argument similar to the one above suffices to prove the statement. The remaining part of the theorem follows immediately.

Corollary 1. Let $G$ be a compact group and let $S$ be an adapted subset of $M^{1}(G)$ such that $S \cap P^{1}(G) \neq \varnothing$. Then there are $\mu_{1}, \ldots, \mu_{n} \in S$ so that for all $1<p<\infty$,

$$
L_{0}^{p}(G)=\left\{\sum_{i=1}^{n}\left(f_{i}-\mu_{i} * f_{i}\right): f_{i} \in L^{p}(G) \text { for } 1 \leq i \leq n\right\} .
$$

Also, any linear functional on $L^{p}(G)$ which is $\mu_{i}$-invariant for all $i, 1 \leq i \leq n$, is a multiple of Haar measure.

Proof. Let $\phi \in S \cap \mathscr{P}^{1}(G)$. Then $\hat{\phi} \in c_{0}(\hat{G})[3$, p. 81]. It is clear from this that Theorem 1 applies.

Corollary 2. Let $G$ be a compact group with a finite number of components, let $1<p<\infty$ and let $\phi \in \mathscr{P}^{1}(G)$ be such that $\int_{C} \phi d \lambda>0$ for each component $C$ of $G$. Then $L_{0}^{p}(G)=\left\{f-\phi * f: f \in L^{p}(G)\right\}$ and every $\phi$-invariant linear functional on $L^{p}(G)$ is a multiple of Haar measure.

Proof. We have, for any Borel subset $B$ of $G$,

$$
\int_{B} \tilde{\phi} * \phi d \lambda=\int_{G}\left(\int_{t^{-1} B} \phi(s) d \lambda(s)\right) \phi\left(t^{-1}\right) d \lambda(t) .
$$

If $\int_{A} \tilde{\phi} * \phi d \lambda=1$, since $\tilde{\phi} * \phi \in \mathscr{P}^{1}(G)$, it follows that $\int_{A \cap C} \tilde{\phi} * \phi d \lambda>0$ for each component $C$ of $G$. In particular, $\int_{A \cap C_{0}} \tilde{\phi} * \phi d \lambda>0$, where $C_{0}$ is the component of the identity. It follows from Proposition 2 of [9] that the group generated by $A \cap C_{0}$ equals $C_{0}$. Hence, the group generated by $A$ contains $C_{0}$ and intersects each coset of $C_{0}$, so it must equal $G$. Hence $\tilde{\phi} * \phi$ is adapted. Now Lemmas 2(ii) and 3 show that Theorem 1 applies with $S=\{\phi\}$.

Corollary 3. Let $G$ be a compact abelian group, and let $\mu \in M^{1}(G)$ be such that $|\hat{\mu}|$ is bounded away from 1 on $\hat{G} \cap\{l\}^{c}$. Then for each $1<p<\infty$,

$$
L_{0}^{p}(G)=\left\{f-\mu * f: f \in L^{p}(G)\right\} .
$$


Any linear functional on $L^{p}(G)$ which is $\mu$-invariant is a multiple of the Haar measure on $G$.

Proof. Since $|\hat{\mu}|$ is bounded away from 1 on $\hat{G} \cap\{l\}^{c}, \mu$ is not supported by a proper closed subgroup of $G$. Hence $\mu$ is adapted and Theorem 1 applies.

Remarks. 1. Corollary 2 implies that if $\phi \in \mathscr{P}^{1}(G)$, where $G$ is a compact connected group, then $L_{0}^{p}(G)=\left\{f-\phi * f: L^{p}(G)\right\}$ for all $1<p<\infty$. This is a substantial strengthening of the Corollary to Theorem 4 in [9], which only applies to such groups when the regular representation of $G$ upon $L_{0}^{2}(G)$ does not weakly contain the trivial representation.

2. Corollary 3 may also be proved by the method given for $L_{0}^{2}(\mathbf{T})$ in the introduction, and then using interpolation theory.

3. Let $H$ be a countably infinite dense subgroup of a compact abelian group $G$. Then the linear space spanned by $\left\{f-\delta_{h} * f: h \in H\right.$ and $\left.f \in L^{p}(G)\right\}$ is not closed in $L_{0}^{p}(G)$ for $1<p<\infty$ [12 and 13, Theorem 15]. However, $\left\{\delta_{h}: h \in H\right\}$ is adapted, so this shows that the condition in Theorem 1 that $\|\hat{\nu}(\sigma)\| \leq \delta<1$ for $\sigma \in \hat{G} \cap F^{c}$ cannot be dropped. On the other hand, as the results mentioned in the introduction concerning group translations show ([6, Theorem 1], for example), this condition is not always essential for a conclusion along the lines of Theorem 1.

\section{RESULTS ON NONCOMPACT GROUPS}

In this section, conditions are given which ensure that certain types of discontinuous invariant functionals exist on various function spaces on noncompact groups. If $A, K$ are relatively compact subsets of $G$, let

$$
Z(A, K)=\left\{x: x \in A \text { and } x A^{-1} \supseteq K\right\} .
$$

Lemma 5. Let $K, C$ be relatively compact subsets of $G$. Then there is a relatively compact open subset $A$ of $G$ such that $A \supseteq C$ and $Z(A, K) \supseteq C$.

Proof. Let $A$ be relatively compact, open and such that $A^{-1} \supseteq C^{-1} K \cup C^{-1}$. Then $A \supseteq C$ and if $x \in C, x A^{-1} \supseteq K$ so that $x \in Z(A, K)$. Hence $Z(A, K) \supseteq C$.

Lemma 6. Let $\mu \in M^{1}(G)$ and let $K$ be a compact subset of $G$ such that $\mu(K)=1$. Let $A$ be a Borel subset of $G$. Then the function $\chi_{A}-\mu * \chi_{A}$ is zero outside of $(A \cup K A) \cap Z(A, K)^{c}$.

Proof. We have $\left(\mu * \chi_{A}\right)(x)=\int_{G} \chi_{A}\left(s^{-1} x\right) d \mu(s)=\int_{K} \chi_{x A^{-1}} d \mu$. Hence $\mu * \chi_{A}=$ 1 on $Z(A, K)$, so $\chi_{A}-\mu * \chi_{A}=0$ on $Z(A, K)$. Also, if $x A^{-1} \cap K=\varnothing$, then $\mu * \chi_{A}(x)=0$. Hence $\chi_{A}-\mu * \chi_{A}=0$ outside of $K A \cup A$. Thus, $\chi_{A}-\mu * \chi_{A}$ is zero outside $(A \cup K A) \cap Z(A, K)^{c}$, as required.

Definitions. If $A$ is a Borel subset of $G$ and $\mu \in M(G)$, let $\mu_{A} \in M(G)$ be given by $\mu_{A}(B)=\mu(A \cap B) \mu(A)^{-1}$, if $\mu(A) \neq 0$, and by $\mu_{A}=0$, if $\mu(A)=0$. Then if $\mu \in M^{1}(G)$ and $\mu(A)>0, \mu_{A} \in M^{1}(G)$. 
When $G$ is a $\sigma$-compact group, let $\mathscr{S}(G)$ be the set of all sequences $\tau=$ $\left(K_{n}\right)_{n=1}^{\infty}$ of relatively compact subsets of $G$ such that:

(i) each set $K_{n}$ is open,

(ii) $K_{n} \subseteq K_{n+1}$ for all $n$,

(iii) $K_{n}=K_{n}^{-1}$ for all $n$,

(iv) $e \in K_{n}$ for all $n$, and

(v) $G=\bigcup_{n=1}^{\infty} K_{n}$.

Since $G$ is $\sigma$-compact, $\mathscr{S}(G) \neq \varnothing$. If $\tau \in \mathscr{S}(G)$, let

$$
M(\tau)=\left\{\mu: \mu \in M^{1}(G) \text { and } \sum_{n=1}^{\infty}\left(1-\mu\left(K_{n}\right)\right)<\infty\right\} .
$$

$M(\tau)$ is a convex subset of $M^{1}(G)$ and $M_{c}^{1}(G) \subseteq M(\tau)$, where $M_{c}^{1}(G)$ denotes the measures in $M^{1}(G)$ of compact support. Hence, $\Delta\left(L^{1}(G), G\right) \subseteq$ $\Delta\left(L^{1}(G), M(\tau)\right)$.

Theorem 2. Let $G$ be $\sigma$-compact but not compact, and let $\tau \in \mathscr{S}(G)$. Then $\Delta\left(L^{1}(G), M(\tau)\right)$ is not closed in $L_{0}^{1}(G)$, and there is a discontinuous linear functional on $L^{1}(G)$ which is $M(\tau)$-invariant.

Proof. Let $\tau=\left(K_{n}\right) \in \mathscr{S}(G)$. Let $L_{n}=G \cap K_{n}^{c}$, for $n \in \mathbb{N}$. Then $\mu=$ $\mu\left(K_{n}\right) \mu_{K_{n}}+\mu\left(L_{n}\right) \mu_{L_{n}}$, for $\mu \in M^{1}(G)$ and $n \in \mathbb{N}$. Let $f \in L^{1}(G)$ and let $A$ be a Borel subset of $G$. Then, for $\mu \in M^{1}(G)$,

$$
\begin{aligned}
\left|\int_{A}(f-\mu * f) d \lambda\right| & \leq\left|\int_{A}\left(f-\mu_{K_{n}} * f\right) d \lambda\right|+\left|\mu\left(L_{n}\right) \int_{A}\left(\mu_{K_{n}} * f-\mu_{L_{n}} * f\right) d \lambda\right| \\
& \leq\left|\int_{G} f\left(\chi_{A}-\tilde{\mu}_{K_{n}} * \chi_{A}\right) d \lambda\right|+2 \mu\left(L_{n}\right)\|f\|_{1} \\
& \leq \int_{T_{n}}|f| d \lambda+2 \mu\left(L_{n}\right)\|f\|_{1},
\end{aligned}
$$

where $T_{n}$ is any Borel subset of $G$ such that $\chi_{A}-\tilde{\mu}_{K_{n}} * \chi_{A}$ is zero outside of $T_{n}$.

Let $V$ be an open relatively compact neighborhood of $e$. Define inductively a sequence of open, relatively compact subsets of $G$ as follows: $A_{1}=V$ and when $A_{1}, \ldots, A_{r}$ have been defined, let $A_{r+1}$ be open, relatively compact and such that $A_{r+1} \supseteq A_{r}, A_{r+1} \cap A_{r}^{c}$ contains some left translate of $V$ and $Z\left(A_{r+1}, K_{r+1}\right) \supseteq K_{r} A_{r}$ (here Lemma 5 has been used).

Let $r \in \mathbb{N}$ and $\mu \in M^{1}(G)$. Put $T_{r}=K_{r} A_{r} \cap Z\left(A_{r}, K_{r}\right)^{c}$. As $e \in K_{r}$ and $K_{r}=K_{r}^{-1}$, we deduce from Lemma 6 that $\chi_{A_{r}}-\tilde{\mu}_{K_{r}} * \chi_{A_{r}}$ is zero outside $T_{r}$. Also, if $r>s, Z\left(A_{r}, K_{r}\right) \supseteq K_{r-1} A_{r-1} \supseteq T_{s}$, so that $T_{r} \cap T_{s}=\varnothing$. Hence, for any $\mu \in M^{1}(G)$, the functions in the sequence $\left(\chi_{A_{n}}-\tilde{\mu}_{K_{n}} * \chi_{A_{n}}\right)_{n=1}^{\infty}$ are concentrated on pairwise disjoint sets. 
Let $f \in \Delta\left(L^{1}(G), M(\tau)\right)$, and let $f_{1}, \ldots, f_{q} \in L^{1}(G), \mu_{1}, \ldots, \mu_{q} \in M(\tau)$ be such that $f=\sum_{i=1}^{q}\left(f_{i}-\mu_{i} * f_{i}\right)$. Let $\left(\mu_{i}\right)_{K_{n}}$ be denote by $\mu_{i, K_{n}}$. We now have, using (4.1),

$$
\begin{aligned}
\left|\int_{A_{n}} f d \lambda\right| & \leq \sum_{i=1}^{q}\left|\int_{A_{n}}\left(f_{i}-\mu_{i} * f_{i}\right) d \lambda\right| \\
& \leq \sum_{i=1}^{q} \int_{T_{n}}\left|f_{i}\right| d \lambda+2 \sum_{i=1}^{q}\left(1-\mu_{i}\left(K_{n}\right)\right)\left\|f_{i}\right\|_{1} .
\end{aligned}
$$

Since the sets in $\left(T_{n}\right)_{n=1}^{\infty}$ are pairwise disjoint and $\sum_{n=1}^{\infty}\left(1-\mu_{i}\left(K_{n}\right)\right)<\infty$ for each $1 \leq i \leq q$, we now have

$$
\begin{aligned}
\sum_{n=1}^{\infty}\left|\int_{A_{n}} f d \lambda\right| & \leq\left(\sum_{i=1}^{q}\left\|f_{i}\right\|_{1}\right)\left(1+2 \max _{1 \leq i \leq q}\left(\sum_{n=1}^{\infty}\left(1-\mu_{i}\left(K_{n}\right)\right)\right)\right) \\
& <\infty, \quad \text { for all } f \in \Delta\left(L^{1}(G), M(\tau)\right) .
\end{aligned}
$$

Since $A_{n+1} \cap A_{n}^{c}$ contains a left translate of $V$, for each $n \in \mathbb{N}$ choose $a_{n} \in G$ so that $a_{n} V \subseteq A_{n} \cap A_{n-1}^{c}$ (if $n=1$ let $a_{1}=e$ and $A_{0}=\varnothing$ ). Then the method of $\left[16\right.$, p. 212] may now be used to construct $\phi \in L_{0}^{1}(G)$ as follows: $\phi(x)=\lambda(V)^{-1}$ for $x \in A_{1}, \phi(x)=-2^{-n} \lambda(V)^{-1}$ for $x \in a_{2^{n}} V$ and $n \geq$ 1 , and $\phi(x)=0$ elsewhere. Then as in [16], $\sum_{n=1}^{\infty}\left|\int_{A_{n}} \phi d \lambda\right|=\infty$, so by (4.2) $\phi \notin \Delta\left(L^{1}(G), M(\tau)\right)$. However, since $\Delta\left(L^{1}(G), G\right) \subseteq \Delta\left(L^{1}(G), M(\tau)\right)$, it follows from Proposition 3(iii) that $\phi \in \overline{\Delta\left(L^{1}(G), M(\tau)\right)}=L_{0}^{1}(G)$. Hence $\Delta\left(L^{1}(G), M(\tau)\right)$ is not closed in $L^{1}(G)$. The proof of the theorem is completed by applying Proposition 1.

Remark. Theorem 2 leaves open the question of whether $\Delta\left(L^{1}(G), M^{1}(G)\right)=$ $L_{0}^{1}(G)$. Equivalently, are there discontinuous $M^{1}(G)$-invariant linear functions on $L^{1}(G)$ ?

Definition. Let $\tau=\left(K_{n}\right) \in \mathscr{S}(G)$ and let $\beta=\left(\beta_{n}\right)$ be a decreasing sequence of strictly positive numbers with limit 0 . Then define $M(\tau, \beta)$ to be the set of all measures $\mu$ in $M^{1}(G)$ such that, for some $K \in \mathbb{R}$ (depending on $\mu$ ), $1-\mu\left(K_{n}\right) \leq K \beta_{n}$, for all $n \in \mathbb{N} . M(\tau, \beta)$ is a convex subset of $M^{1}(G)$ which contains $M_{c}^{1}(G)$. The following is an analogue for $L^{p}(G) \quad(1<p \leq \infty)$ of Theorem 2 which applied for $L^{1}(G)$.

Theorem 3. Let $G$ be noncompact, $\sigma$-compact and amenable. Let $\tau=\left(K_{n}\right) \in$ $\mathscr{S}(G)$ and let $\beta=\left(\beta_{n}\right)$ be a decreasing sequence of strictly positive numbers whose limit is 0 . Then the following hold:

(i) If $1<r<p<\infty$ and $\beta \in l^{r}, \Delta\left(L^{p}(G), M(\tau, \beta)\right)$ is not closed in $L^{p}(G)$, and there is a discontinuous linear functional on $L^{p}(G)$ which is $M(\tau, \beta)$-invariant, and 
(ii) if $X$ denotes any one of the spaces $C_{0}(G), C U(G), C(G), L^{\infty}(G)$, then $\Delta(X, M(\tau, \beta))$ is not closed in $X$ and there is a discontinuous, $M(\tau, \beta)$-invariant functional on $X$.

Proof. Let $1<r<p \leq \infty$ and $p^{-1}+q^{-1}=1$. Let $\delta>0$ be chosen so that $r / p+\delta<1$ (if $p=\infty, q=1$ and $r / p=0$ ). As $G$ is amenable, there is a sequence $\left(V_{n}\right)$ of open, relatively compact subsets of $G$ so that $K_{n} \subseteq V_{n} \subseteq V_{n+1}$ and $\lambda\left(s^{-1} V_{n} \Delta V_{n}\right)<\beta_{n}^{(r / p+\delta) q} \lambda\left(V_{n}\right)$, for all $n \in \mathbb{N}$ and $s \in K_{n}$.

Now if $f \in L^{p}(G)$ and $\mu \in M^{1}(G)$, a calculation along the lines of [16, $\mathrm{p}$. 209] shows that

$$
\left|\int_{V_{n}}\left(f-\mu_{K_{n}} * f\right) d \lambda\right| \leq\|f\|_{p} \beta_{n}^{r / s+\delta} \lambda\left(V_{n}\right)^{1 / q} .
$$

Also, if $\mu \in M(\tau, \beta)$ and $M>0$ is chosen so that $1-\mu\left(K_{n}\right) \leq M \beta_{n}$ for all $n$, and if we use the fact that $\mu=\mu\left(K_{n}\right) \mu_{K_{n}}+\left(1-\mu\left(K_{n}\right)\right) \mu_{G \cap K_{n}^{c}}$ (as in the proof of Theorem 2), we find that

$$
\left|\int_{V_{n}}(f-\mu * f) d \lambda\right| \leq\|f\|_{p} \beta_{n}^{r / p+\delta} \lambda\left(V_{n}\right)^{1 / q}\left\{1+2 M \beta_{n}^{1-(r / p+\delta)}\right\} .
$$

It follows that if $\mu \in M(\tau, \beta)$, there is a constant $M^{\prime}$ so that

$$
\left|\int_{V_{n}}(f-\mu * f) d \lambda\right| \leq M^{\prime}\|f\|_{p} \beta_{n}^{r / p+\delta} \lambda\left(V_{n}\right)^{1 / q}, \quad \text { for all } n \in \mathbb{N} \text { and } f \in L^{p}(G) \text {. }
$$

An easy consequence of this is that if $h \in \Delta\left(L^{p}(G), M(\tau, \beta)\right)$, there is $L>0$ so that

$$
\left|\int_{V_{n}} h d \lambda\right| \leq L \beta_{n}^{r / p+\delta} \lambda\left(V_{n}\right)^{1 / q}, \text { for all } n .
$$

Now consider the case where $1<r<p<\infty$ and $\beta \in l^{r}$. Define $g$ on $G$ by $g(x)=\beta_{1}^{r / p} \lambda\left(V_{1}\right)^{-1 / p}$ for $x \in V_{1}$, and $g(x)=\beta_{n}^{r / p} \lambda\left(V_{n}\right)^{-1 / p}$ for $x \in V_{n} \cap V_{n-1}^{c}$ and $n \geq 2$. Then by a similar argument to [16, p. 210], it follows that $g \in L^{p}(G)$, so that

$$
\left|\int_{V_{n}} g d \lambda\right| \geq \beta_{n}^{r / p} \lambda\left(V_{n}\right)^{1 / q}, \quad \text { for all } n .
$$

Comparing this with the preceding inequality, we see that

$$
g \notin \Delta\left(L^{p}(G), M(\tau, \beta)\right) .
$$

Since $\Delta\left(L^{p}(G), M(\tau, \beta)\right)$ is dense in $L^{p}(G)$ by Proposition 3(ii), we see that $\Delta\left(L^{p}(G), M(\tau, \beta)\right)$ is not closed, and the rest of (i) follows from Proposition 1 .

In this case where $p=\infty$, choose a decreasing sequence $\left(\theta_{n}\right)$ of strictly positive numbers so that $\lim _{n \rightarrow \infty} \theta_{n}^{-1} \beta_{n}^{\delta}=0$. Let $U$ be a symmetric, open, 
relatively compact neighborhood of $e$. Define $\psi(x)=\theta_{1}$ for $x \in U V_{1}$, and $\psi(x)=\theta_{n}$ for $x \in U V_{n} \cap U V_{n-1}^{c}$ and $n \geq 2$. Let $g=\lambda(U)^{-1} \chi_{U}$ and define $\phi \in C_{0}(G)$ by putting $\phi=g * \psi$. Similarly to [16, p. 210], $\left|\int_{V_{n}} \phi d \lambda\right| \geq \theta_{n} \lambda\left(V_{n}\right)$, and by (4.3) it follows that $\phi \notin \Delta\left(L^{\infty}(G), M(\tau, \beta)\right)$. Part (ii) of the Theorem now follows easily using Proposition 1 and Proposition 3(iv).

Remarks. The proofs of Theorems 2 and 3 are based upon the approach taken by G. Woodward in proving corresponding results for the spaces $\Delta\left(L^{p}(G), G\right)$ [16, Theorems 1 and 2]. In the case of Theorem 3, substantial modification was required to the proof of Theorem 1 in [16], so a relatively detailed proof has been presented. In the case of Theorem 3, lesser modification was required, so the proof of Theorem 3 has been somewhat truncated.

2. Theorem 3 leaves it open as to whether there are discontinuous $M^{1}(G)$ invariant linear functionals on $L^{p}(G)$, for $1<p<\infty$.

Lemma 7. Let $g$ be a nonzero, nonnegative measurable function on $\mathbb{R}$ which is integrable on each compact subset of $\mathbb{R}$ and vanishes on $(-\infty, 0)$. Let $a>0$ and let $\psi \in \mathscr{P}^{1}(\mathbb{R})$ be such that for some $b \in \mathbb{R}, \psi=0$ on $(-\infty, b)$. Then the following hold:

(i) if $b \geq 0, \int_{-a}^{a}(\psi * g)(t) d t \leq \int_{-a}^{a} g(t) d t$, and

(ii) $\psi * g=0$ on $(-\infty, b)$.

Proof. Part (i) follows easily from the fact that

$$
\int_{-a}^{a}(\psi * g)(t) d t=\int_{b}^{\infty} \psi(s)\left[\int_{-s-a}^{-s+a} g(t) d t\right] d s
$$

and (ii) follows from the definition of convolution.

Theorem 4. Let $X$ denote any one of the Banach spaces $L^{p}(\mathbb{R})$ for $1 \leq p \leq \infty$, $C_{0}(\mathbb{R}), C U(\mathbb{R}), C(\mathbb{R})$ or $L^{\infty}(\mathbb{R})$. Then $\Delta(X, \mathbb{R})$ is a subspace of $\Delta\left(X, \mathscr{P}^{1}(\mathbb{R})\right)$ and it has infinite codimension in $\Delta\left(X, \mathscr{P}^{1}(\mathbb{R})\right)$. There exists in $X^{\prime}$ an infinite, linearly independent family of translation invariant linear functionals, such that each of these is discontinuous and is not $\mathscr{P}^{1}(\mathbb{R})$-invariant.

Proof. The Corollary to Proposition 2 shows that $\Delta(X, \mathbb{R})$ is a subspace of $\Delta\left(X, \mathscr{P}^{1}(\mathbb{R})\right)$. If $n, s \in \mathbb{N}$ let $K_{n, s}=\left(-n^{s}, n^{s}\right)$ and let $\tau_{s}=\left(K_{n, s}\right)_{n=1}^{\infty} \in$ $\mathscr{S}(\mathbb{R})$. Also, let $V=(-1,1)$.

Case 1. $1<p \leq \infty$. Let $1<r<p$, let $\delta>0$ be chosen so that $r / p+\delta<1$, and let $p^{-1}+q^{-1}=1$ (if $p=\infty, 0<\delta<1, r / p=0$ and $q=1$ ). If $n, s \in \mathbb{N}$ let $V_{n, s}=\left(-c_{n, s}, c_{n, s}\right)$, where $c_{n, s}=n^{(r / p+\delta) q+s}$. Finally, let $\beta=\left(\beta_{n}\right)_{n=1}^{\infty}$ where $\beta_{n}=n^{-1}$ for all $n$.

It is easy to check that $K_{n, s} \subseteq V_{n, s}$ and that

$$
\lambda\left(\left(-t+V_{n, s}\right) \Delta V_{n, s}\right) \leq \beta_{n}^{(r / p+\delta) q} \lambda\left(V_{n, s}\right)
$$


for all $n \in \mathbb{N}$ and $t \in K_{n, s}$. It follows now from (4.3) in the proof of Theorem 3 that if $X$ is any one of the given spaces except $L^{1}(\mathbb{R})$, for each $h \in \Delta\left(X, M\left(\tau_{s}, \beta\right)\right)$ there is $L>0$ so that

$$
\left|\int_{V_{n, s}} h d \lambda\right| \leq L n^{s / q}, \quad \text { for all } n \in \mathbb{N} .
$$

Now for $n, s \in \mathbb{N}$ let $\phi_{n, s}$ and $\phi_{s}$ be given in $\mathscr{P}^{1}(\mathbb{R})$ by

$$
\phi_{n, s}=\chi_{\left(c_{n, s}, c_{n, s}+1\right)} \text { and } \phi_{s}=(\zeta(1+\delta / 2))^{-1} \sum_{i=1}^{\infty} i^{-(1+\delta / 2)} \phi_{i, s} .
$$

Let $g$ be any function as in Lemma 7. Applying this lemma, and observing that $c_{i, s} \geq c_{n, s}$ if and only if $i \geq n$ now gives

$$
\begin{aligned}
\int_{V_{n, s}}\left(g-\phi_{s} * g\right) d \lambda & =(\zeta(1+\delta / 2))^{-1} \sum_{i=1}^{\infty} i^{-(1+\delta / 2)} \int_{-c_{n, s}}^{c_{n, s}}\left(g-\phi_{i, s} * g\right)(t) d t \\
& \geq(\zeta(1+\delta / 2))^{-1}\left(\sum_{i=n}^{\infty} i^{-(1+\delta / 2)}\right)\left(\int_{0}^{c_{n, s}} g(t) d t\right) \\
& \geq(\zeta(1+\delta / 2))^{-1} 2 \delta^{-1} n^{-\delta / 2} \int_{0}^{c_{n, s}} g(t) d t .
\end{aligned}
$$

Case IA. $1<p<\infty$ and $X=L^{p}(\mathbb{R})$. For $s \in \mathbb{N}$ define $g_{s}$ on $\mathbb{R}$ by letting

$$
\begin{aligned}
& g_{s}(x)=1, \quad \text { for } x \in[0,1), \\
& g_{s}(x)=n^{-r / p} c_{n, s}^{-1 / p}, \quad \text { for } x \in\left[c_{n-1, s}, c_{n, s}\right), n \geq 2, \quad \text { and } \\
& g_{s}(x)=0, \quad \text { for } x \in(-\infty, 0) .
\end{aligned}
$$

Then $g_{s} \in L^{p}(\mathbb{R})$ since

$$
\begin{aligned}
\int_{\mathbb{R}}\left|g_{s}\right|^{p} d \lambda & =\sum_{n=1}^{\infty} n^{-r} c_{n, s}^{-1}\left(c_{n, s}-c_{n-1, s}\right) \\
& \leq \sum_{n=1}^{\infty} n^{-r}<\infty, \quad \text { as } r>1 .
\end{aligned}
$$

Also,

$$
\int_{V_{n, s}} g_{s} d \lambda \geq n^{-r / p} c_{n, s}^{-1 / p} c_{n, s}=n^{\delta+s / q}
$$

It now follows from (4.5) that for all $n \in \mathbb{N}$

$$
\int_{V_{n, s}}\left(g_{s}-\phi_{s} * g_{s}\right) d \lambda \geq(\zeta(1+\delta / 2))^{-1} 2 \delta^{-1} n^{\delta / 2+s / q}
$$

Comparing this with (4.4) shows that for each $s \in \mathbb{N}$,

$$
g_{s}-\phi_{s} * g_{s} \notin \Delta\left(L^{p}(\mathbb{R}), M\left(\tau_{s}, \beta\right)\right) \text {. }
$$


On the other hand, if $s_{1}, s_{2} \in \mathbb{N}$, a routine calculation shows that $\phi_{s_{1}} \in$ $M\left(\tau_{s_{2}}, \beta\right)$ if and only if $s_{2} \geq 2 \delta^{-1}\left(s_{1}+(r / p+\delta) q\right)$.

Now let $s_{1} \in \mathbb{N}$ and define $s_{n} \in \mathbb{N}$ by letting $s_{n}>2 \delta^{-1}\left(s_{n-1}+(r / p+\delta) q\right)$ for $n \geq 2$. Then the subspaces $\Delta\left(L^{p}(\mathbb{R}), M\left(\tau_{s_{n}}, \beta\right)\right)$ of $L^{p}(\mathbb{R})$ increase as $n$ increases. Also, for all $n \in \mathbb{N}$,

$$
g_{s_{n}}-\phi_{s_{n}} * g_{s_{n}} \in \Delta\left(L^{p}(\mathbb{R}), M\left(\tau_{s_{n+1}}, \beta\right)\right) \cap\left(\Delta\left(L^{p}(\mathbb{R}), M\left(\tau_{s_{n}}, \beta\right)\right)\right)^{c} .
$$

It follows easily from these facts that no nontrivial linear combination of functions in $\left\{g_{s_{n}}-\phi_{s_{n}} * g_{s_{n}}: n \in \mathbb{N}\right\}$ belongs to $\Delta\left(L^{p}(\mathbb{R}), M\left(\tau_{s_{1}}, \beta\right)\right)$ or to $\Delta\left(L^{p}(\mathbb{R}), \mathbb{R}\right)$, since this latter subspace is smaller. Since each function $g_{s_{n}}-\phi_{s_{n}} * g_{s_{n}} \in \Delta\left(L^{p}(\mathbb{R}), \mathscr{P}^{1}(\mathbb{R})\right)$, it follows that $\Delta\left(L^{p}(\mathbb{R}), \mathbb{R}\right)$ has infinite codimension in $\Delta\left(L^{p}(\mathbb{R}), \mathscr{P}^{1}(\mathbb{R})\right)$. For each $n \in \mathbb{N}$, there is $L_{n} \in\left(L^{p}(\mathbb{R})\right)^{\prime}$ so that $L_{n}=0$ on $\Delta\left(L^{p}(\mathbb{R}), \mathbb{R}\right), L_{n}\left(g_{s_{n}}-\phi_{s_{n}} * g_{s_{n}}\right)=1$ and $L_{n}\left(g_{s_{m}}-\phi_{s_{m}} * g_{s_{m}}\right)=0$ if $m \neq n$. Then $\left\{L_{n}: n \in \mathbb{N}\right\}$ is an independent family of translation invariant functionals which are not $\mathscr{P}^{1}(G)$-invariant. Proposition 3(ii) implies that each $L_{n}$ is discontinuous. This proves the theorem when $X=L^{p}(\mathbb{R})$ and $1<p<\infty$.

Case IB. $p=\infty$ and $X=C_{0}(\mathbb{R}), C U(\mathbb{R}), C(\mathbb{R})$ or $L^{\infty}(\mathbb{R})$. In this case let $h=2^{-1} \chi_{V}=2^{-1} \chi_{(-1,1)} \in \mathscr{P}^{1}(\mathbb{R})$. Note that in this case $c_{n, s}=n^{\delta+s}$. If $s \in \mathbb{N}$ define $\psi_{s}$ on $\mathbb{R}$ by letting

$$
\begin{aligned}
& \psi_{s}(x)=0, \quad \text { if } x \in(-\infty, 1), \quad \text { and } \\
& \psi_{s}(x)=n^{-\delta / 3}, \quad \text { if } x \in\left[(n-1)^{\delta+s}+1, n^{\delta+s}+1\right), n \in \mathbb{N} .
\end{aligned}
$$

Then $h * \psi_{s} \in C_{0}(\mathbb{R}), h * \psi_{s}=0$ on $(-\infty, 0]$, and $\left(h * \psi_{s}\right)(x) \geq n^{-\delta / 3}$, for $x \in\left[2, n^{\delta+s}\right)$. If we now use the approach in the preceding case with $h * \psi_{s}$ in place of $g_{s}$ we find that

$$
\begin{aligned}
& \int_{V_{n, s}}\left(h * \psi_{s}-\phi_{s} * h * \psi_{s}\right) d \lambda \geq(\zeta(1+\delta / 2))^{-1} 2 \delta^{-1} n^{-\delta / 2} \int_{0}^{n^{s+\delta}}\left(h * \psi_{s}\right)(t) d t \\
& \quad \geq(\zeta(1+\delta / 2))^{-1} 2 \delta^{-1} n^{-\delta / 2}\left\{\int_{0}^{2}\left(h * \psi_{s}\right)(t) d t+\int_{2}^{n^{s+\delta}}\left(h * \psi_{s}\right)(t) d t\right\} \\
& \quad \geq(\zeta(1+\delta / 2))^{-1} 2 \delta^{-1} n^{-\delta / 2}\left(n^{s+\delta}-2\right) n^{-\delta / 3} \\
& \quad \geq(\zeta(1+\delta / 2))^{-1} \delta^{-1} n^{s+\delta / 6}, \quad \text { if } n \geq 4^{1 /(s+\delta)} .
\end{aligned}
$$

Comparing this with (4.4) with $q=1$ shows that $h * \psi_{s}-\phi_{s} * h * \psi_{s} \notin$ $\Delta\left(L^{\infty}(\mathbb{R}), M\left(\tau_{s}, \beta\right)\right)$. The statements in the theorem for this case now follow by an argument similar to the one for $L^{p}(\mathbb{R}), 1<p<\infty$.

Case II. $p=1$ and $X=L^{1}(\mathbb{R})$. If $n, s \in \mathbb{N}$ let $A_{n, s}=\left(-n^{s+1}, n^{s+1}\right)$. Then $V=A_{1}^{s}, A_{r+1, s} \cap A_{r, s}^{c}$ contains a translate of $V$ and $Z\left(A_{r+1, s}, K_{r+1, s}\right) \supseteq$ $A_{r, s}+K_{r, s}$. It follows from (4.2) in the proof of Theorem 2 that 


$$
\sum_{n=1}^{\infty}\left|\int_{A_{n, s}} f d \lambda\right|<\infty \text { for all } f \in \Delta\left(L^{1}(\mathbb{R}), M\left(\tau_{s}\right)\right) \text {. }
$$

Now let $\theta_{s}=(\zeta(2))^{-1} \sum_{i=1}^{\infty} i^{-2} \chi_{\left(i^{s+1}, i^{s+1}+1\right)} \in \mathscr{P}^{1}(\mathbb{R})$, and let $h \in L^{1}(\mathbb{R})$ be such that $h \geq 0, h=0$ on $(-\infty, 0)$, and $h \neq 0$. We deduce from Lemma 7 and a similar argument to the one used in the previous cases that for all $n \in \mathbb{N}$,

$$
\int_{-n^{s+1}}^{n^{s+1}}\left(h-\theta_{s} * h\right)(t) d t \geq \zeta(2)^{-1} n^{-1} \int_{0}^{n^{s+1}} h(t) d t .
$$

Consequently,

$$
\sum_{n=1}^{\infty}\left|\int_{A_{n, s}}\left(h-\theta_{s} * h\right) d \lambda\right|=\infty
$$

so that by (4.6) above, $h-\theta_{s} * h \notin \Delta\left(L^{1}(\mathbb{R}), M\left(\tau_{s}\right)\right)$. On the other hand, it is routine to check that $\theta_{s_{1}} \in M\left(\tau_{s_{2}}\right)$ if and only if $s_{2}>s_{1}+1$. Hence $h-\theta_{2 s} * h \in \Delta\left(L^{1}(\mathbb{R}), M\left(\tau_{2 s+2}\right)\right) \cap\left(\Delta\left(L^{1}(\mathbb{R}), M\left(\tau_{2 s}\right)\right)\right)^{c}$, for all $s \in \mathbb{N}$. The conclusion of the theorem for $X=L^{1}(\mathbb{R})$ now follows along similar lines to the previous cases.

Remark. When $G$ is a noncompact, nondiscrete, $\sigma$-compact group which is amenable as a discrete group, it has been proved by E. Granirer [2] (see also related results of W. Rudin in [14] that $L^{\infty}(G)$ has an invariant mean which is not $\mathscr{P}^{1}(G)$-invariant. Corresponding results for $C(G)$ have been derived by $\mathrm{J}$. Rosenblatt [11]. The means on these spaces are continuous. Theorem 4 seems to provide the first example of a discontinuous linear functional on a space $L^{\infty}(G)$ or $C(G)$ which is translation invariant but not $\mathscr{P}^{1}(G)$-invariant.

\section{REFERENCES}

1. J. Bourgain, Translation invariant forms on $L^{p}(G)(1<p<\infty)$, Ann. Inst. Fourier (Grenoble) 36 (1986), 97-104.

2. E. Granirer, Criteria for compactness and for discreteness of locally compact amenable groups, Proc. Amer. Math. Soc. 40 (1973), 615-624.

3. E. Hewitt and K. A. Ross, Abstract harmonic analysis. Vol. II, Springer-Verlag, 1970.

4. B. E. Johnson, $A$ proof of the translation invariant form conjecture for $L^{2}(G)$, Bull. Sci. Math. (2) 197 (1983), 301-310.

5. Y. Katznelson, An introduction to harmonic analysis, Wiley, New York, 1968.

6. G. H. Meisters and W. M. Schmidt, Translation-invariant linear forms on $L^{2}(G)$ for compact abelian groups $G$, J. Funct. Anal. 11 (1972), 407-424.

7. G. H. Meisters, Some discontinuous translation-invariant linear forms, J. Funct. Anal. 12 (1973), 199-210.

8. __ Some problems and results on translation-invariant linear forms, Radical Banach Algebras and Automatic Continuity, (J. M. Bachar and W. G. Bade et al., Eds.), Lecture Notes in Math., vol. 975, Springer-Verlag, 1983.

9. R. Nillsen, Group actions and direct sum decompositions of $L^{p}$ spaces, Proc. Amer. Math. Soc. 106 (1989), 975-985. 
10. J.-P. Pier, Amenable locally compact groups, Wiley, New York, 1984.

11. J. Rosenblatt, Invariant means on the continuous bounded functions, Trans. Amer. Math. Soc. 236 (1978), 315-324.

12. $\ldots$, Translation invariant linear forms on $L^{p}(G)$, Proc. Amer. Math. Soc. 94 (1985), 226-228.

13. __ Ergodic group actions, Arch. Math. 47 (1986), 263-269.

14. W. Rudin, Invariant means on $L^{\infty}$, Studia Math. 44 (1972), 219-227.

15. G. A. Willis, Continuity of translation invariant linear functionals on $C_{0}(G)$ for certain locally compact groups $G$, Monatsh. Math. 105 (1988), 161-164.

16. G. Woodward, Translation-invariant linear forms on $C_{0}(G), C(G), L^{p}(G)$ for noncompact groups, J. Funct. Anal. 12 (1973), 205-220.

Department of Mathematics, University of Wollongong, Wollongong, New South WALES, 2500, Australia 